\begin{tabular}{|c|c|}
\hline Title & A n error evaluation scheme based on rotation of magnetic field in adaptive finite element analysis \\
\hline Author(s) & Matsutomo, Shinya; Miyamoto, Tomoyuki; Kaneda, Kazufumi; Noguchi, So; Y amashita, Hideo \\
\hline Citation & $\begin{array}{l}\text { IEEE transaction on magnetics, 42(4), 567-570 } \\
\text { https://doi.org/10.1109/T MA G.2006.871627 }\end{array}$ \\
\hline Issue Date & $2006-04$ \\
\hline Doc URL & http:/hdl .handle.net/2115/8521 \\
\hline Rights & $\begin{array}{l}\text { (c) } 2006 \text { IEEE. Personal use of this material is permitted. However, permission to reprint/republish this material for } \\
\text { advertising or promotional purposes or for creating new collective works for resale or redistribution to servers or lists, } \\
\text { or to reuse any copyrighted component of this work in other works must be obtained from the }\end{array}$ \\
\hline Type & article \\
\hline File Information & 01608269.pdf \\
\hline
\end{tabular}

Instructions for use 


\title{
An Error Evaluation Scheme Based on Rotation of Magnetic Field in Adaptive Finite Element Analysis
}

\author{
Shinya Matsutomo ${ }^{1}$, Tomoyuki Miyamoto ${ }^{2}$, Kazufumi Kaneda ${ }^{2}$, So Noguchi ${ }^{3}$, and Hideo Yamashita ${ }^{4}$ \\ ${ }^{1}$ Niihama National College of Technology, Ehime 792-8580, Japan \\ ${ }^{2}$ Graduate School of Engineering, Hiroshima University, Hiroshima 739-8527, Japan \\ ${ }^{3}$ Graduate School of Information Science and Technique Hokkaido University, Sapporo 060-0814, Japan \\ ${ }^{4}$ Graduate School of Engineering, Hiroshima Institute of Technology, Hiroshima 731-5193, Japan
}

\begin{abstract}
The finite-element analysis is widely used in design stage of electromagnetic apparatuses. The analysis accuracy depends on the characteristics of the finite-element mesh, e.g., number of nodes, number of elements and shape of elements. Recently, the adaptive finite-element analysis is one of the most promising numerical analysis techniques. In process of the adaptive finite-element method, the error evaluation is one of the important schemes. In this paper, a new error evaluation scheme, which is suitable for electromagnetic problems, is proposed. The proposed error evaluation method is then applied to two-dimensional and three-dimensional magnetostatic field problems for its verification.
\end{abstract}

Index Terms-Adaptive finite-element analysis, adaptive meshing, electromagnetic problem, error evaluation.

\section{INTRODUCTION}

I N ELECTROMAGNETIC field analysis, the finite-element method (FEM) is widely used as a numerical analysis technique. Commonly, for using the FEM, a user has to make a finiteelement mesh suitable for highly accurate analysis and less computation time. However, making such a suitable mesh is a bottleneck to the easy and wide use of the FEM, since the mesh-making work is time-consuming. Moreover, the analysis accuracy and the computation time depend on the mesh characteristics, e.g., number of nodes, number of elements, and shape of elements, which require some experience for the user to make a suitable mesh. Therefore, the adaptive FEM [1]-[4] is one of the promising numerical analysis techniques for users without experiment and even with enough experiences. By applying the adaptive FEM, the mesh is automatically and appropriately refined, and produces results whose error is less than the permissible error. The adaptive FEM has three important schemes: true value estimation scheme, error evaluation scheme and mesh control scheme.

In this paper, a new error evaluation scheme based on rotation of the magnetic field is proposed. In the newly proposed method, the error evaluation is calculated by simple equations, which are derived from the field governing equations. Then we have applied the proposed error evaluation scheme to 2-D and 3-D magnetostatic field problems for its verification.

\section{Proposed Error Evaluation Method}

\section{A. Error Evaluation Scheme}

In this paper, we focus our attention on the governing equations in magnetostatic field problem, as follows:

$$
\begin{aligned}
\operatorname{rot}(\nu \operatorname{rot} \boldsymbol{A}) & =\boldsymbol{J} \\
\boldsymbol{B} & =\operatorname{rot} \boldsymbol{A} \\
\boldsymbol{H} & =\nu \boldsymbol{B}
\end{aligned}
$$

where $\nu, A, J, B$ and $\boldsymbol{H}$ are the reluctivity, the magnetic vector potential, the current density, the magnetic flux density and the intensity of magnetic field, respectively.

Here, the newly proposed error evaluation method based on rotation of magnetic field is described. In the finite-element analysis for magnetostatic field problems, $\boldsymbol{B}^{e}$ and $\boldsymbol{H}^{e}$ ( $e$ denotes element) are obtained. Here, $\boldsymbol{B}^{e}$ and $\boldsymbol{H}^{e}$ should be satisfied with (1), as follows:

$$
\operatorname{rot}\left(\boldsymbol{B}^{e}\right)=\mu \boldsymbol{J}^{e}, \text { or } \operatorname{rot}\left(\boldsymbol{H}^{e}\right)=\boldsymbol{J}^{e}
$$

where $\mu$ is the permeability. For evaluating the error of $\boldsymbol{B}^{e}$ and $\boldsymbol{H}^{e}$, it is necessary to estimate a more accurate value for $\boldsymbol{B}^{e}$ and $\boldsymbol{H}^{e}$. In this paper, the estimation process is called the true value estimation. After the true value estimation, the more accurately estimated values, $\overline{\boldsymbol{B}}^{e}$ and $\overline{\boldsymbol{H}}^{e}$ (which are calculated from the neighboring elements, as will be described in the next session), are obtained. These values are not strictly satisfied with (4). Therefore, we propose two error evaluation schemes using the integral of residuals, as follows:

$$
\begin{aligned}
& \varepsilon_{\boldsymbol{B}}^{e}=\int\left|\operatorname{rot} \overline{\boldsymbol{B}^{e}}-\mu \boldsymbol{J}^{e}\right| \mathrm{d} S^{e}=\int\left|\operatorname{rot}\left(\mu \overline{\boldsymbol{H}^{e}}\right)-\mu \boldsymbol{J}^{e}\right| \mathrm{d} S^{e} \\
& \varepsilon_{\boldsymbol{H}}^{e}=\int\left|\operatorname{rot} \overline{\boldsymbol{H}^{e}}-\boldsymbol{J}^{e}\right| \mathrm{d} S^{e}
\end{aligned}
$$

where $\varepsilon_{\boldsymbol{B}}^{\varepsilon}$ and $\varepsilon_{\boldsymbol{H}}^{\varepsilon}$ represent the evaluated error on element $e$.

The two error evaluation methods presented above are essentially similar, since (5) and (6) are based on rotation of the magnetic field. However, (5) is different from (6) in the permeability. That is, the estimated error in (5) is weighted by means of the rotation of the magnetic field times its permeability. By adopting (6), consequently, the elements in the high permeability region, e.g., iron, tends to be refined. On the other hand, by adopting (5), the elements in the high magnetic field regions on the air, e.g., air-gap, tends to be refined.

In this paper, we compare the above error estimation methods and the Zienkiewicz-Zhu (Z-Z) method [5], which was proposed 
for elastic problem. However, the Z-Z method is widely employed for the electromagnetic problems [3] [4], and in commercial software. The Z-Z method is based on energy error calculated from

$$
\begin{aligned}
\lambda_{e}=\left[\frac{1}{2} \int\left(\boldsymbol{B}^{e}-\overline{\boldsymbol{B}}^{e}\right) \cdot \nu\left(\boldsymbol{B}^{e}-\overline{\boldsymbol{B}}^{e}\right) \mathrm{d} S^{e}\right] \\
\quad\left[\frac{1}{2} \int \overline{\boldsymbol{B}}^{e} \cdot \nu \overline{\boldsymbol{B}}^{e} \mathrm{~d} S^{e}\right]
\end{aligned}
$$

where $\lambda_{e}$ represents the evaluated error on element $e$.

\section{B. True Value Estimation Scheme}

In order to evaluate the error of the analysis result, it is necessary to estimate the more accurate value, which is as close to the true value as possible. Here, the way to estimate such accurate value of the magnetic flux density, $\overline{\boldsymbol{B}}^{e}$, and the intensity of magnetic field, $\overline{\boldsymbol{H}}^{e}$, obtained from the FEM result with coarser mesh, are presented.

The magnetic flux density, $\boldsymbol{B}^{e}$, and the intensity of magnetic field, $\boldsymbol{H}^{e}$, are constant on each element when the linear element is employed. Before the true value estimation regarding to $\boldsymbol{B}^{e}$ and $\boldsymbol{H}^{e}$ on element $e, \boldsymbol{B}_{i}$ and $\boldsymbol{H}_{i}$ at node $i$ are estimated from the neighboring elements connecting to $i$, by calculating

$$
\boldsymbol{B}_{i}=\left[\sum_{j=1}^{n} w_{j}^{e} \boldsymbol{B}_{j}^{e}\right] /\left[\sum_{j=1}^{n} w_{j}^{e}\right]
$$

and

$$
\boldsymbol{H}_{i}=\left[\sum_{j=1}^{n} w_{j}^{e} \boldsymbol{H}_{j}^{e}\right] /\left[\sum_{j=1}^{n} w_{j}^{e}\right]
$$

where $n$ is the number of the elements connecting to node $i, w_{j}^{e}$ is the weight for element $j$. The weight values can be determined according to the area or the angle of the connecting elements. However, we have investigated that the weight doesn't give a large influence to the accuracy of the estimated true value [6]. In this paper, hence, the value of the weight, $w^{e}$, is "1" for all elements. After the estimation about $\boldsymbol{B}_{i}$ and $\boldsymbol{H}_{i}$ at node $i, \overline{\boldsymbol{B}}^{e}$ and $\overline{\boldsymbol{H}}^{e}$ on element $e$ are calculated by simply interpolated from $\boldsymbol{B}_{i}$ and $\boldsymbol{H}_{i}$.

However, when the node $i$ is on the surface of materials with the different magnetic permeabilities, $\boldsymbol{B}_{i}$ is separately calculated for each material. For example, in Fig. 1, the node $i$ is on the interface between two different materials, material- 1 and -2 . The magnetic flux density $\boldsymbol{B}_{\boldsymbol{i}}$ as to material-1 is calculated from that of the neighboring elements 1 to $n$ in material- 1 as a more accurate value. The $\boldsymbol{B}_{i}$ in material-2 is calculated from the elements $n+1$ to $m$. Thereby, there are two values of the magnetic flux density at the node $i$. On the other hand, $\boldsymbol{H}_{i}$ is calculable regardless of the magnetic permeability. As a more accurate value, therefore, the intensity of the magnetic field $\left(\boldsymbol{H}_{i}\right)$ at the node $i$ is simply calculated form the elements 1 to $m$ in Fig. 1 .

\section{Verification IN 2-D Magnetostatic Field Problem}

The adaptive FEM with the newly proposed error evaluation methods based on the rotation of $\boldsymbol{B}$ and $\boldsymbol{H}$, i.e., (5) and (6), respectively, is applied to a 2 -D linear magnetostatic field

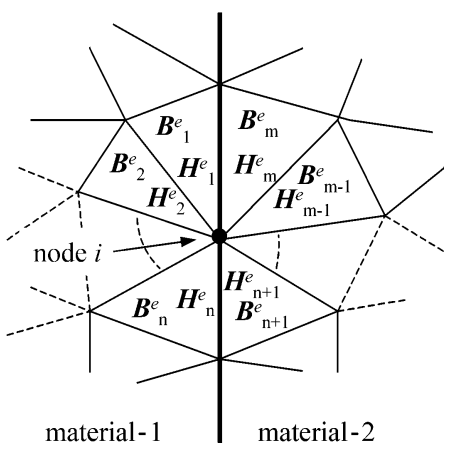

Fig. 1. True value estimation of magnetic flux density at node $i$ on the interface between material-1 and material-2 with different magnetic permeabilities.

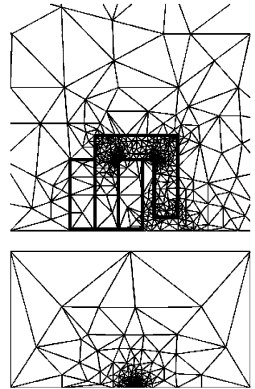

(a)

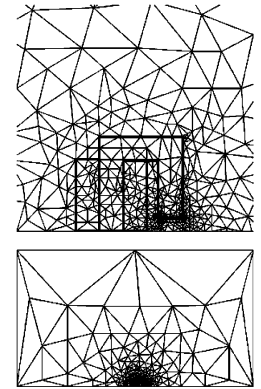

(b)

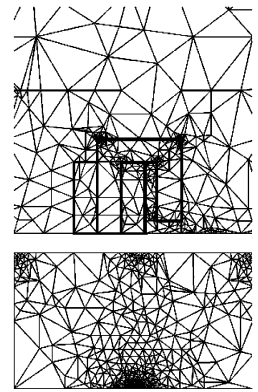

(c)
Fig. 2. Final finite-element meshes obtained for 2-D analysis. The upper figures represent the expanded view of iron and coil. The lower figures represent the whole analysis region. (a) Rotation $B$, (b) rotation $H$, and (c) $\mathrm{Z}-\mathrm{Z}$ method.

problem and the results are compared with that of the Z-Z method [5]. The test model consists of an iron core with air gap and coils, as shown in Fig. 2. For the verification, the accuracies regarding to the coil inductance error and the magnetic flux density error were compared with the highly accurate value, which is acquired with very fine mesh. As a mesh refinement, it is adopted that elements evaluated with high errors values are simply divided in two. The comparison is done with the number of elements generated by each error evaluation method being approximately the same. The comparison results are shown in Table I. The meshes resulting from the adaptive FEMs are shown in Fig. 2. As a result, we can see that the proposed method provides high accuracy and generates the suitable mesh for magnetostatic field problem.

\section{Application for 3-D MAgnetostatic Field PRoblem}

\section{A. Applied Model and Mesh Refinement Scheme}

For a further verification of the usability, the adaptive finiteelement method with the newly proposed method is applied to a 3-D linear magnetostatic field problem, using tetrahedral finiteelement mesh. The test model consists of an iron core and coils, and it is an eighth part of region, as shown in Fig. 3.

As a mesh refinement scheme, in this paper, the dynamic bubble mesh generation system [7] is employed to remesh according to the proposed error evaluation. The dynamic bubble mesh generation system produces the tetrahedral mesh according to the bubble radii, which are set at all the corners of materials in the analysis region, as shown in Fig. 4. In the adaptive FEM, the size of bubble radii is suitably decided according 
TABLE I

RESUlts OF ADAPTIVE FINITE-ELEMENT METHODS

\begin{tabular}{c|ccc}
\hline Error evaluation method & rotation $\boldsymbol{B}$ & rotation $\boldsymbol{H}$ & $\mathrm{Z}-\mathrm{Z}$ \\
\hline Coil inductance error [\%] & 3.50 & 2.00 & 5.17 \\
Magnetic flux density error [\%] & 7.08 & 6.54 & 11.20 \\
\hline
\end{tabular}

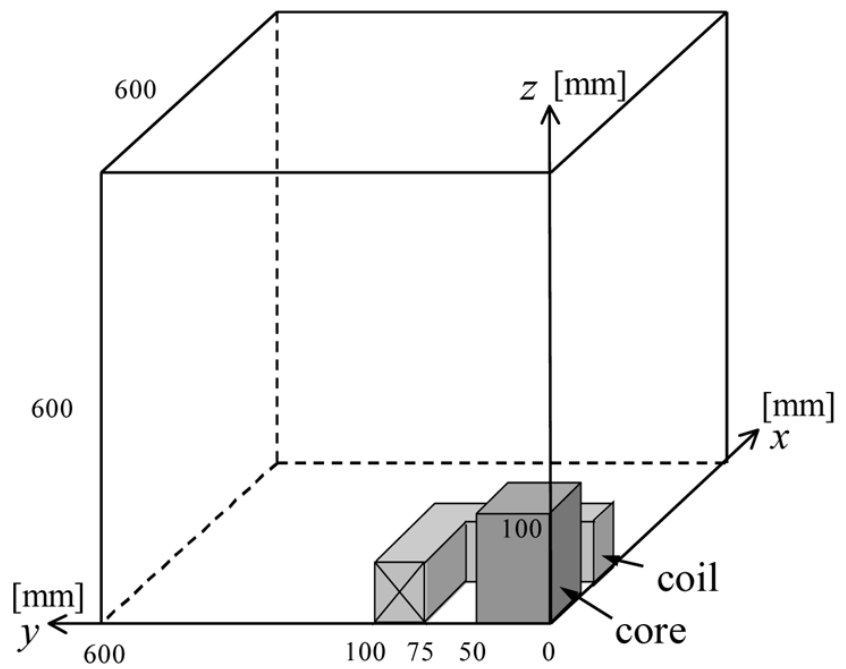

Fig. 3. Three-dimensional analysis model.

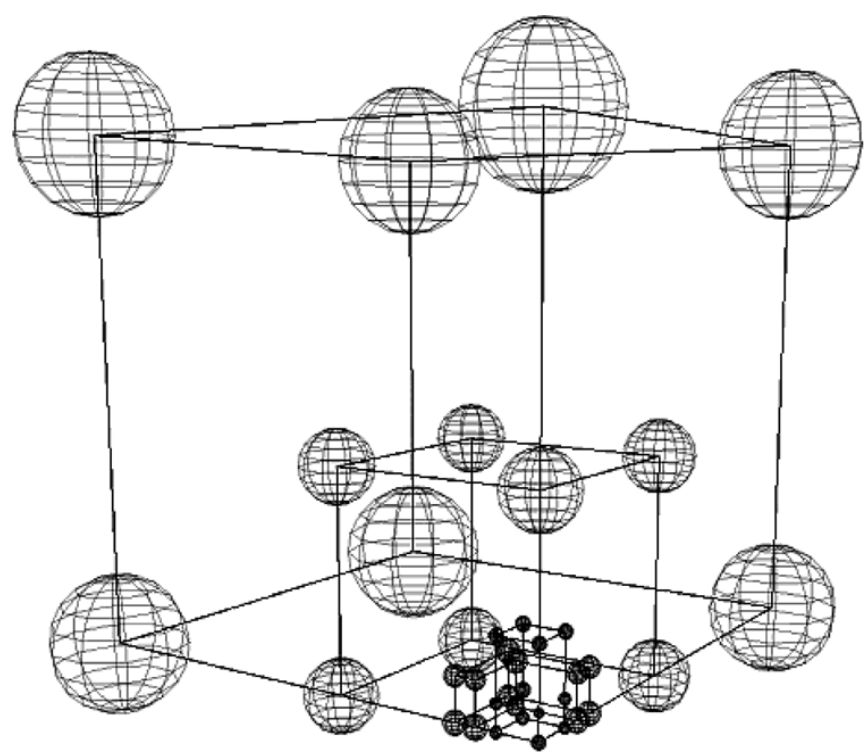

Fig. 4. Bubble radii are set at the corners of the materials in the analysis region.

to the error evaluation scheme, i.e., a large bubble radius is set at the point where a large error is presumed, oppositely, a small bubble radius is set at the point of a small error presumed.

\section{B. Results and Discussion}

In the verification for 3-D magnetostatic field problem, the newly proposed methods (based on the rotation of $\boldsymbol{B}$ and based on the rotation of $\boldsymbol{H}$ ) and the Z-Z method [5] (the methods defined by (5)-(7), respectively), are compared. To each error evaluation method, the same initial coarse mesh is used, and then a suitable fine mesh is generated by applying the adaptive

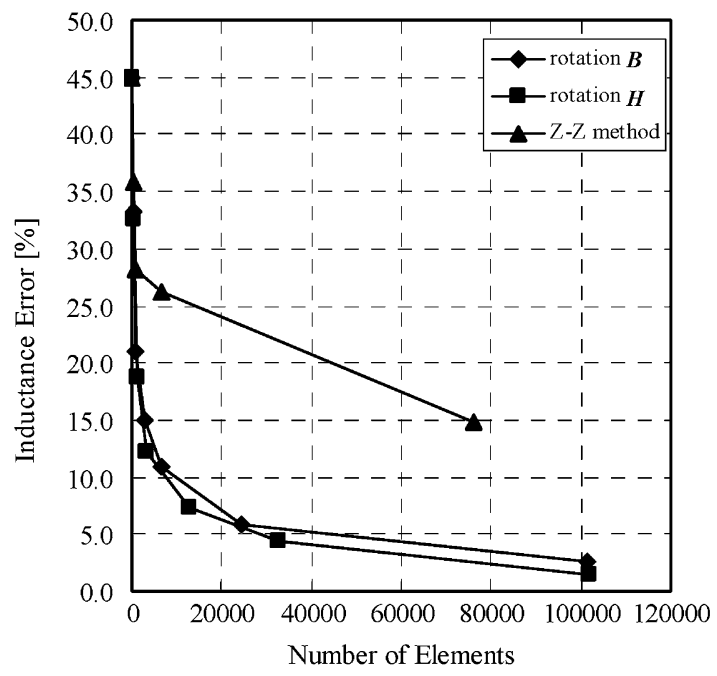

(a)

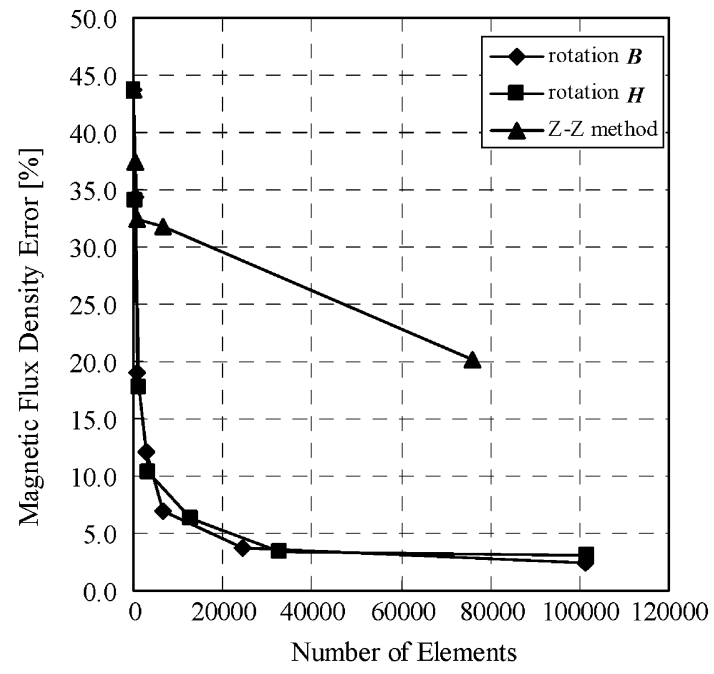

(b)

Fig. 5. Error characteristics as number of elements increases. (a) Coil inductance error versus number of elements. (b) Magnetic flux density error versus number of elements.

FEMs. The accuracies regarding to the coil inductance error and the magnetic flux density error were compared with the highly accurate value, which is acquired with a very fine mesh. The comparison is done under the almost same number of elements generated by each error evaluation method. The comparison results are shown in Fig. 5. The meshes resulting from the adaptive FEMs are shown in Fig. 6.

As a result, the proposed methods provide high accuracies and generate suitable meshes for the 3-D magnetostatic field problem. By applying the newly proposed method based on the rotation of $\boldsymbol{B}$, an excellent mesh as to the magnetic flux density error tends to be obtained. By the newly proposed method based on the rotation of $\boldsymbol{H}$, an excellent mesh as to the coil inductance error tends to be obtained. By the Z-Z method, an excellent result is not obtained for any of these performance parameters. This result is probably due to the fact that the Z-Z method was proposed for elastic analysis, and generates elements that are extremely refined in the area where the magnetic flux density and the energy are low, and the magnetic vector potential is nearly 

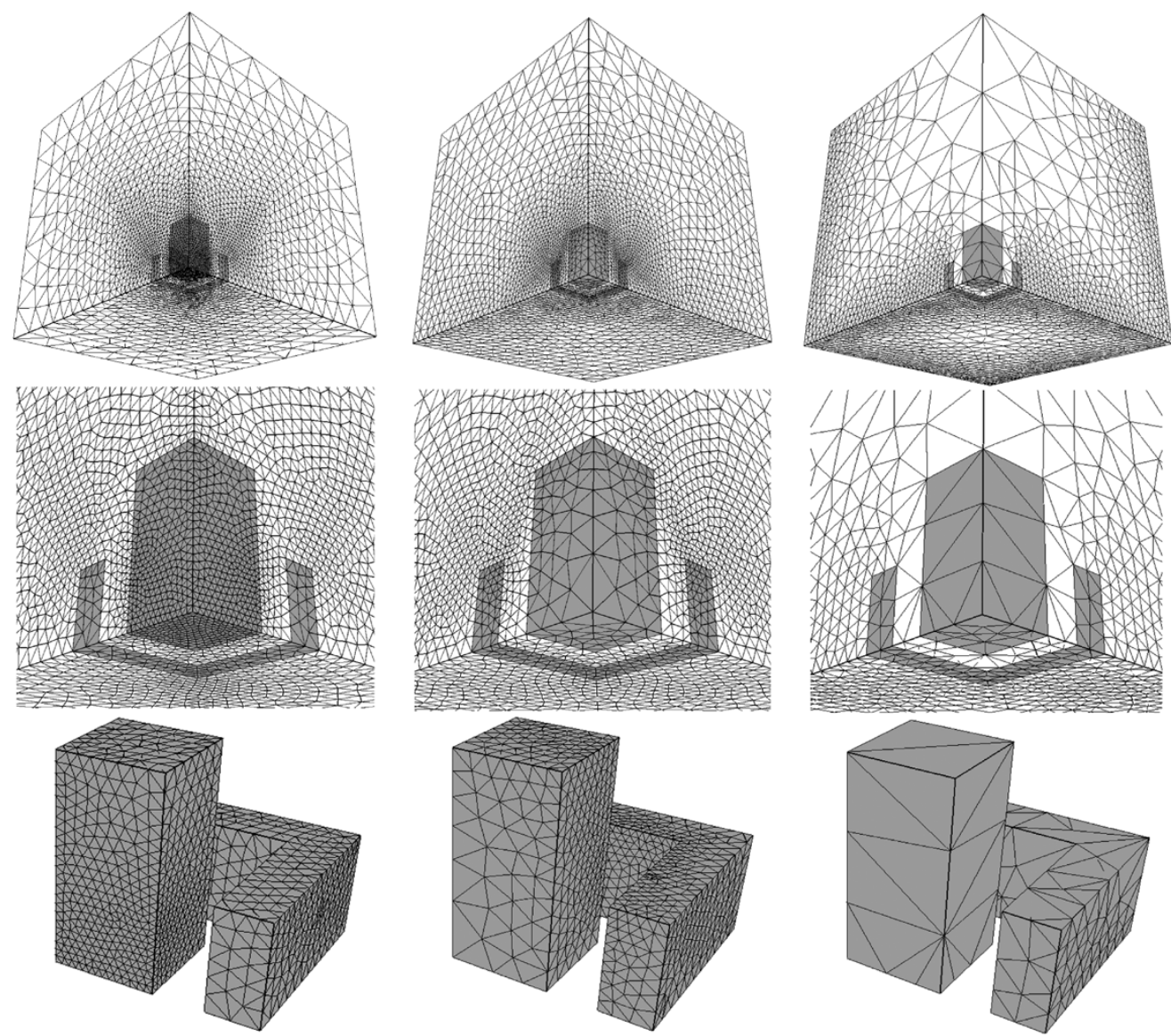

(a)

(b)

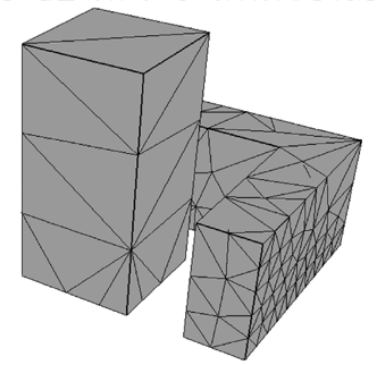

(c)

Fig. 6. Final meshes obtained by each error estimation method. The upper figures represent the whole analysis region. The middle figures are the expanded view of iron and coil. The lower figures represent only iron and coil from another view. (a) Rotation $B$, (b) rotation $H$, and (c) Z-Z method.

" 0 "' as shown in Fig. 6(c). Moreover, the elements of the iron core and the coil aren't small enough.

Additionally, as a final result, very absorbing meshes were obtained by the proposed methods. In the newly proposed method based on the rotation of $\boldsymbol{B}$, the small elements are generated in the region that the magnetic permeability is large and the magnetic flux density drastically changes (e.g., in the iron core), because the magnetic permeability is included in (6) as a weight value. In the newly proposed method based on the rotation of $\boldsymbol{H}$, the small elements are generated in the region that the intensity of magnetic field drastically changes, e.g., around the coil and the end of the iron core.

In the future, another error evaluation should be developed by inheriting the good characteristics from both the newly proposed methods. As the permeability in (6) actually works as a weight to refinement especially for iron, the better mesh will be generated by adjusting the weight instead of the permeability.

\section{CONCLUSION}

In this paper, we have proposed and verified two error evaluation methods based, respectively, on the magnetic flux density and on the magnetic field intensity. The proposed methods provide higher accuracy and generate more suitable meshes for 2-D and 3-D magnetostatic field problems, when compared with the
Zienkiewicz-Zhu method. In the near future, another error evaluation scheme should be developed, by inheriting the good characteristics from both the newly proposed methods.

\section{REFERENCES}

[1] Y. Q. Tan and Y. P. Wu, "An adaptive finite element computation of h-p version for magnetic field problems," IEEE Trans. Magn., vol. 30, no. 5, pp. 3519-3522, Sep. 1994.

[2] L. Janicke and A. Kost, "Error estimation and adaptive mesh generation in the 2D and 3D finite element method," IEEE Trans. Magn., vol. 32, no. 3, pp. 1334-1337, May 1996.

[3] K. Yamazaki, S. Watari, and A. Egawa, "Adaptive finite element meshing for eddy current analysis of moving conductor,' IEEE Trans. Magn., vol. 40, no. 2, pp. 993-996, Mar. 2004.

[4] M. Miwa, D. Dibben, and T. Yamada, "High accuracy torque calculation for a rotating machine using adaptive meshing," IEEE Trans. Magn., vol. 40, no. 2, pp. 1001-1004, Mar. 2004.

[5] O. C. Zienkiewicz and J. Z. Zhu, "A simple error estimate and adaptive procedure for practical engineering analysis," Int. J. Numer. Meth. Eng., vol. 24, pp. 337-357, 1987.

[6] S. Matsutomo, S. Noguchi, and H. Yamashita, "A study on error estimation method for electromagnetic problems in adaptive finite element analysis," in Proc. Joint Technical Meeting Static Apparatus and Rotating Machinery Inst. Elect. Eng. Japan, 2004, pp. 17-22. Paper: SA-04-47/RM-04-71, (in Japanese).

[7] T. Yokoyama, V. Cingoski, K. Kaneda, and H. Yamashita, "3-D automatic mesh Generation for FEA using dynamic bubble system," IEEE Trans. Magn., vol. 35, no. 3, pp. 1318-1321, Mar. 1999.

Manuscript received June 20, 2005 (e-mail: shin@ect.niihama-nct.ac.jp). 